\title{
Dynamique fluviale et évolution des berges du cours inférieur des rivières Nottaway, Broadback et de Rupert, en Jamésie (Québec
}

\author{
Fluvial dynamic and bank evolution of Nottaway, Broadback \\ and Rupert rivers, James Bay area (Québec). \\ Fluss-Dynamik und Entwiclung der Flussufer im unteren Lauf \\ der Flüsse Nottaway, Broadback und Rupert, James Bay Gebiet \\ (Québec).
}

\section{Diane Saint-Laurent et Pierre Guimont}

Volume 53, numéro 3, 1999

URI : https://id.erudit.org/iderudit/004866ar

DOI : https://doi.org/10.7202/004866ar

\section{Aller au sommaire du numéro}

\section{Éditeur(s)}

Les Presses de l'Université de Montréal

ISSN

0705-7199 (imprimé)

1492-143X (numérique)

Découvrir la revue

Citer cet article

Saint-Laurent, D. \& Guimont, P. (1999). Dynamique fluviale et évolution des berges du cours inférieur des rivières Nottaway, Broadback et de Rupert, en Jamésie (Québec. Géographie physique et Quaternaire, 53(3), 389-399.

https://doi.org/10.7202/004866ar

\section{Résumé de l'article}

La dynamique fluviale et l'évolution des berges des rivières Nottaway, Broadback et de Rupert sont examinées en regard des phénomènes d'érosion et des caractéristiques morpho-sédimentologiques. Parmi les nombreux agents d'érosion fluviale, ce sont les vagues et les courants qui sont les plus efficaces. Les berges constituées de matériaux limono-argileux et sableux, dont les pentes sont abruptes $\left(<25^{\circ}\right)$, sont particulièrement sensibles à l'activité fluviale. Les phénomènes et les formes d'érosion les plus fréquemment observés dans la portion aval des rivières étudiées sont les glissements et les éboulements. Ces mouvements de masse entraînent le long des pentes une perte importante de matériaux qui sont directement livrés aux cours d'eau. Les sédiments les plus fins sont transportés en aval et une grande quantité est déversée dans la baie de Rupert. On évalue à près de 883000 tonnes par année la quantité de sédiments en suspension transportés par les rivières Nottaway, Broadback et de Rupert. Ces apports sédimentaires ont pour conséquence de combler progressivement la baie de Rupert qui est déjà peu profonde (3 à $6 \mathrm{~m}$ en moyenne). Ce comblement progressif risque de modifier les conditions hydrologiques et sédimentologiques, notamment en ce qui à trait à l'écoulement et à la circulation des eaux de la baie de Rupert et de la baie de James. Le relèvement isostatique résiduel constitue un autre facteur contribuant à la modification des conditions biophysiques de la baie de Rupert.
Tous droits réservés @ Les Presses de l'Université de Montréal,1999
Ce document est protégé par la loi sur le droit d'auteur. L’utilisation des services d'Érudit (y compris la reproduction) est assujettie à sa politique d'utilisation que vous pouvez consulter en ligne.

https://apropos.erudit.org/fr/usagers/politique-dutilisation/ 


\section{DYNAMIQUE FLUVIALE ET ÉVOLUTION DES BERGES DU COURS INFÉRIEUR DES RIVIËRES NOTTAWAY, BROADBACK ET DE RUPERT, EN JAMÉSIE (QUÉBEC)}

Diane SAINT-LAURENT* et Pierre GUIMONT**, Département de géographie, Université du Québec à Montréal, Montréal, Québec H3C 3P8 et Unité Environnement, Hydro-Québec, 800, boulevard de Maisonneuve Est, Montréal, Québec H2L 4M8.

RÉSUMÉ Dynamique fluviale et évolution des berges du cours inférieur des rivières Nottaway, Broadback et de Rupert, Jamésie (Québec). La dynamique fluviale et l'évolution des berges des rivières Nottaway, Broadback et de Rupert sont examinées en regard des phénomènes d'érosion et des caractéristiques morpho-sédimentologiques. Parmi les nombreux agents d'érosion fluviale, ce sont les vagues et les courants qui sont les plus efficaces. Les berges constituées de matériaux limono-argileux et sableux, dont les pentes sont abruptes $\left(>25^{\circ}\right)$, sont particulièrement sensibles à l'activité fluviale. Les phénomènes et les formes d'érosion les plus fréquemment observés dans la portion aval des rivières étudiées sont les glissements et les éboulements. Ces mouvements de masse entraînent le long des pentes une perte importante de matériaux qui sont directement livrés aux cours d'eau. Les sédiments les plus fins sont transportés en aval et une grande quantité est déversée dans la baie de Rupert. On évalue à près de 883000 tonnes par année la quantité de sédiments en suspension transportés par les rivières Nottaway, Broadback et de Rupert. Ces apports sédimentaires ont pour conséquence de combler progressivement la baie de Rupert qui est déjà peu profonde ( 3 à $6 \mathrm{~m}$ en moyenne). $\mathrm{Ce}$ comblement progressif risque de modifier les conditions hydrologiques et sédimentologiques, notamment en ce qui à trait à l'écoulement et à la circulation des eaux de la baie de Rupert et de la baie de James. Le relèvement isostatique résiduel constitue un autre facteur contribuant à la modification des conditions biophysiques de la baie de Rupert.
ABSTRACT Fluvial dynamic and bank evolution of Nottaway, Broadback and Rupert rivers, James Bay area (Québec). The fluvial dynamic and bank evolution of Nottaway, Broadback and Rupert rivers will be examined in terms of erosion phenomena and morphosedimentary patterns. Based on facts from geomorphology and sedimentology studies as well as field observations, the main factors of erosion and cyclic processes responsible for bank evolution are identified. Among well known factors pertaining fluvial erosion, waves and currents appear to be the most effective agents. Some types of banks are most sensitive than others. Clay banks with a high degree of declivity $\left(>25^{\circ}\right)$ are especially affected by fluvial activity. The most often encountered erosion phenomena and erosion forms in the downstream portions of the three studied rivers are slumps and landslips. Those mass movements are responsible for important loss of material from river banks which is rapidly carried away by the river. The finest particles will be transported downstream up to the Baie de Rupert. Near 883000 tons of suspended matters are transported yearly by Nottaway, Broadback and Rupert rivers to the bay. Those sedimentary loads contribute to the filling of this large basin already as shallow an average of 3 to $6 \mathrm{~m}$ in certain parts. This progressive filling may eventually modify hydrologic and sedimentologic conditions, particularly the water circulation between Rupert and James bays. The residual isostatic rebound must be also considered as a factor of biophysics transformation of the Bay.
ZUSAMMENFASSUNG Fluss-Dynamik und Entwiclung der Flussufer im unteren Lauf der Flüsse Nottaway, Broadback und Rupert, James Bay Gebiet (Québec).Wir haben die Flussdynamik und die Entwicklung der Ufer der Flüsse Nottaway, Broadback und Rupert im Hinblick auf Erosions-Phänomene und morpho-sedimentäre Charakteristika untersucht. Von den zahlreichen Faktoren, welche die Flusserosion bewirken, sind die Wellen und Strömungen die wirksamsten. Die aus Schlamm, Ton und Sand bestehenden Flussufer, deren Böschungen steil sind $\left(>25^{\circ}\right)$, reagieren besonders empfindlich auf die Flussaktivität. Die am häufigsten beobachteten Phänomene und Erosionsformen im unteren Lauf der untersuchten Flüsse sind Abrutschungen und Abbrüche. Diese Massenbewegungen entlang der Böschungen führen zu einem bedeutenden Verlust von Material, welches direkt von den Wasserläufen fortgetragen wird. Die feinsten Sedimente werden flussabwärts transportiert und ein großer Teil davon in die Baie de Rupert. Man schätzt die Menge der Suspensionssedimente, welche die Flüsse Nottaway, Broadback und Rupert transportieren, jährlich auf ungefähr 883000 Tonnen. Diese Sedimentzufuhren füllen allmählich die ohnehin wenig tiefe (durchschnittlich 3-5 m) Baie de Rupert aus. Diese progressive Ausfüllung könnte die hydrologischen und sedimentären Bedingungen verändern, insbesondere den Abfluss und die Zirkulation des Wassers zwischen der Baie de Rupert und der Baie de James. Die restliche isostatische Hebung ist ein weiterer Faktor, der zur Veränderung der biophysischen Bedingungen der Baie de Rupert beiträgt.

\footnotetext{
Manuscrit reçu le 15 décembre 1998 ; manuscrit révisé accepté le 8 juillet 1999

* Adresse électronique : saint-laurent.diane@uqam.ca

** Adresse électronique : guimont.pierre@hydro.qc.ca
} 


\section{INTRODUCTION}

Les rivières Nottaway, Broadback et de Rupert (Complexe NBR) ont fait l'objet de nombreux travaux depuis 1964, notamment dans le cadre des études préliminaires des projets d'aménagements hydroélectriques réalisées par Hydro-Québec (SEBJ, 1982, 1984; Hydro-Québec, 1990). Les plus récentes études concernant les aspects géomorphologiques et morpho-sédimentologiques des berges de ces rivières ont été effectuées entre les années 1989 et 1992 (Sogeam-Tecsult Inc., 1991, 1992 ; Lupien, Rosenberg et Associés, 1991, 1992 ; Hydrotech Inc., 1988, 1991). Elles ont permis de mieux comprendre la dynamique fluviale et son évolution en considérant la nature des sédiments qui composent les berges et en identifiant les principaux agents d'érosion qui les atteignent. Ces études ont aussi fourni des données sur les taux d'érosion et les bilans sédimentaires des rivières en cause.

La photo-interprétation, l'analyse et la compilation des données de terrain ont permis de dresser une cartographie détaillée des berges en considérant leurs caractéristiques morpho-sédimentologiques (composition des matériaux, hauteur et degré des pentes, etc.). On constate que certains types de berges sont généralement plus touchées par l'érosion fluviale que d'autres, notamment les berges aux pentes abruptes constituées de matériaux argileux ou limono-argileux. Ce type de berges domine d'ailleurs presque tout le tronçon aval des rivières Nottaway, Broadback et de Rupert. La présente contribution fait la synthèse des données recueillies lors de plusieurs campagnes de terrain en considérant les formes d'érosion, la nature et la géomorphologie des berges, les bilans sédimentaires et les taux d'érosion du cours inférieur des rivières à l'étude.

\section{SITUATION GÉOGRAPHIQUE ET CONTEXTE GÉOMORPHOLOGIQUE}

Les rivières Nottaway, Broadback et de Rupert occupent le versant oriental du Territoire de la Baie de James (fig. 1). Elles se jettent dans la baie de Rupert qui, elle-même, se déverse vers le nord dans la baie de James. La région étudiée est caractérisée principalement par une plaine argileuse et un plateau rocheux situé à quelque $160 \mathrm{~km}$ à l'est de la côte. Sa limite nord est contiguë au territoire du complexe La Grande et elle est délimitée au sud par les régions de l'Abitibi et du Lac-Saint-Jean. Cette région comprend trois grandes unités physiographiques : 1) la plaine argileuse située en bordure de la côte et qui se prolonge vers le sud dans l'axe des lacs Evans, Matagami et Waswanipi, caractérisée par un relief plat recouvert de grandes tourbières ; 2) la zone de till du centre-nord, caractérisée par la présence de petits massifs rocheux recouverts de dépôts morainiques discontinus ; 3) les collines rocheuses à l'est qui forment un relief légèrement ondulé, dont les dénivelés varient entre 10 et $30 \mathrm{~m}$. Une mince couverture sédimentaire discontinue de till orienté et de platières sableuses s'associent aux petits massifs rocheux (SEBJ, 1982 ; Hydro-Québec, 1990).
Les formes et dépôts tardi-holocènes dans la région témoignent des événements géologiques survenus au cours des derniers millénaires, dont les plus marquants ont été la déglaciation et la formation du Lac glaciaire Barlow-Ojibway, suivies de récurrences glaciaires et de la transgression de la Mer de Tyrrell. La déglaciation du territoire s'est effectuée du sud vers le nord, puis vers le nord-est (Vincent, 1989 ; Dyke et al., 1989). Le Lac Barlow-Ojibway s'est formé par l'endiguement des eaux entre le front glaciaire au nord et la ligne de partage des eaux au sud, vers 9000 ans BP (Hardy, 1977). Les eaux glacio-lacustres ont inondé les basses terres situées à l'est de la côte jamésienne jusqu'à la Moraine de Sakami et, au nord, jusqu'à proximité du site actuel des villages de Kuujjuarapik et de Whapmagoostui. Après le retrait des glaces et le drainage du Lac Ojibway (vers 7900 ans BP), les eaux de la Mer de Tyrrell ont envahi les basses terres du territoire (fig. 1), soit les régions à l'ouest de la moraine de Sakami. La limite marine aurait alors atteint les altitudes approximatives de $200 \mathrm{~m}$ le long de la rivière Nottaway, de $250 \mathrm{~m}$ sur l'interfluve des rivières BroadbackRupert et un maximum de $290 \mathrm{~m}$ au nord de la rivière de Rupert. La transgression marine aurait favorisé la sédimentation de particules fines en eau profonde, de sable silteux en eau moins profonde et de dépôts littoraux grossiers sur les rivages soumis à l'action des vagues et des glaces. Des relevés effectués dans la partie ouest de la région, notamment à l'embouchure de la rivière de Rupert, révèlent d'importantes accumulations d'argile marine (près de $23 \mathrm{~m}$ ) (Sogeam, 1980a).

\section{CARACTÉRISTIQUES GÉNÉRALES DES BASSINS VERSANTS}

Les bassins versants des rivières Nottaway, Broadback et de Rupert couvrent une superficie d'environ $130000 \mathrm{~km}^{2}$ (Hydro-Québec, 1990). Les nombreux cours d'eau de cette région (fig. 1) contribuent à augmenter la charge sédimentaire des trois principales rivières qui alimentent en sédiments fins la baie de Rupert. On évalue à 882625 t/a la quantité de sédiments en suspension déversés dans cette baie auxquels s'ajoutent 188750 t/a de matériaux transportés par charriage, soit un total de 1071375 t/a (Hydrotech Inc., 1991). La rivière Nottaway fournit à elle seule une charge sédimentaire de 590817 t/a, ce qui représente près de $60 \%$ de la charge des rivières du complexe NottawayBroadback-Rupert.

L'ensemble du réseau hydrographique de la rivière Nottaway draine une superficie d'environ 65800 km² (HydroQuébec, 1990). Son immense bassin comporte de nombreux affluents dont les rivières Bell et Waswanipi qui se déversent dans les lacs Matagami et Soscumica, au nord. Ces lacs sont eux-mêmes alimentés par de nombreux tributaires. À sa sortie du lac Soscumica, la rivière Nottaway traverse la plaine argileuse sur tout son parcours, avant de rejoindre la baie de Rupert. La rivière Broadback, pour sa part, draine une superficie d'environ 20860 km² (HydroQuébec, 1990), soit moins de la moitié de celle du bassin de la rivière de Rupert et moins du tiers du bassin de la rivière 


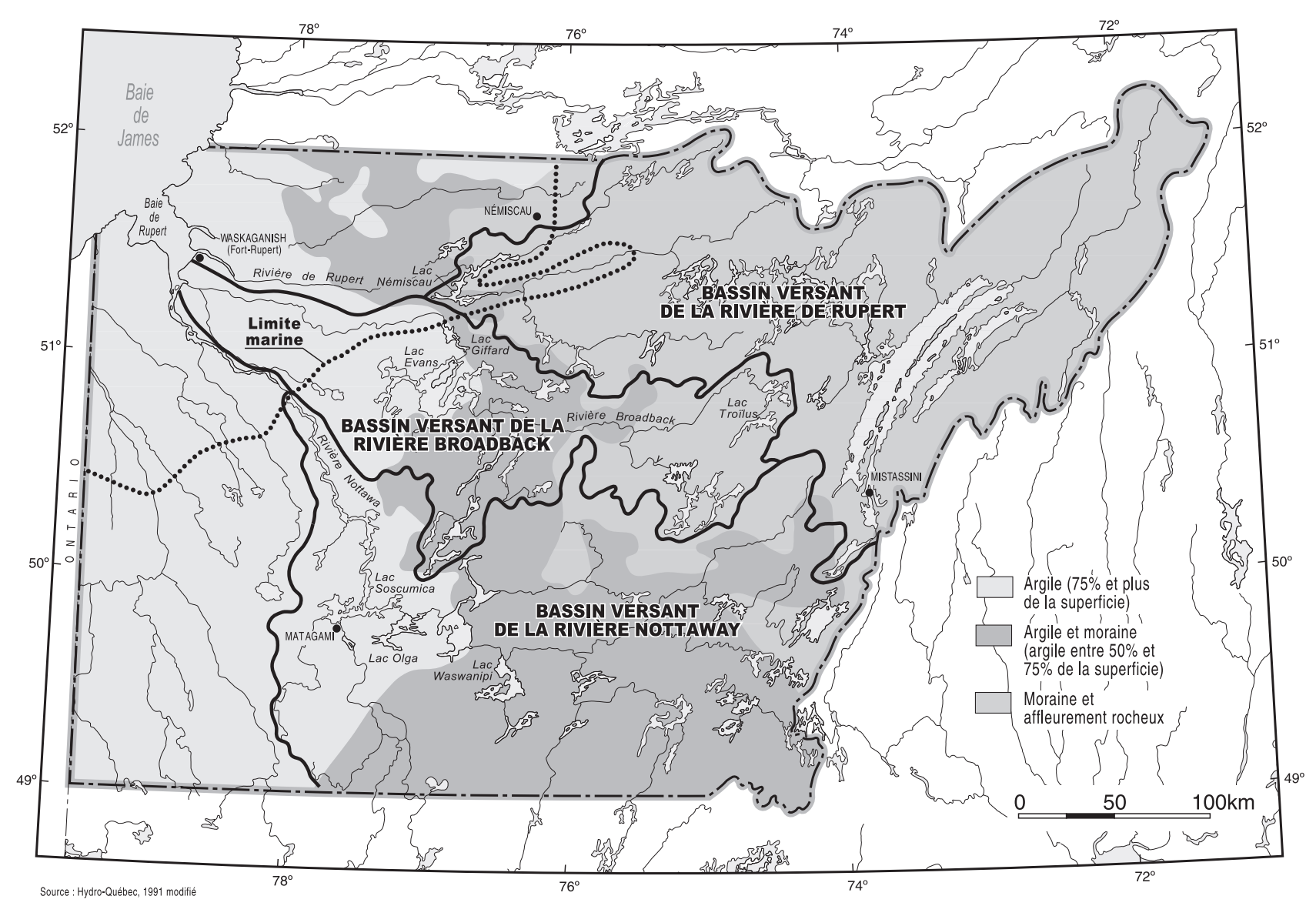

FIGURE 1. Carte de localisation des bassins versants des rivières Nottaway, Broadback et de Rupert, en Jamésie.

Nottaway. Prenant sa source dans le lac Troïlus, la rivière Broadback s'écoule en direction de l'ouest, et traverse la zone des collines rocheuses et la zone des grands lacs Quénonisca et Evans jusqu'au lac Giffard. Son parcours, orienté d'est en ouest, se poursuit dans la plaine côtière jusqu'à son embouchure. De son point de départ à son point d'arrivée, la rivière franchit une distance de $435 \mathrm{~km}$. Les apports de sédiments (suspension et charriage) déversés dans la baie de Rupert par la rivière Broadback sont évalués, en moyenne, à 144783 t/a (Hydrotech Inc., 1991). La rivière de Rupert est la deuxième rivière en importance du complexe NBR. Elle draine un bassin versant de $43253 \mathrm{~km}^{2}$ et circule d'est en ouest sur une distance de 740 km (Hydro-Québec, 1990). Émissaire du lac Mistassini, la rivière traverse successivement la zone de collines rocheuses, la zone de till profilé, ainsi que la plaine argileuse. Les charges sédimentaires qu'elle transporte sont évaluées en moyenne à 265775 t/a à son embouchure (Hydrotech Inc., 1991).

Le transport des sédiments vers la baie de Rupert se fait principalement en mai et en juin, au moment où le débit des rivières est à son maximum (Hydrotech Inc., 1988, 1991). On évalue que plus de la moitié (soit $54 \%$ ) des apports déversés dans la baie surviennent durant cette période, $21 \%$ pour la période estivale et $25 \%$ pour le reste de
Location map of catchment basins for Nottaway, Broadback and Rupert rivers, James Bay area.

l'année (Hydrotech Inc., 1988, 1991). Les rivières Nottaway, Broadback et de Rupert ont un régime d'écoulement pluvionival marqué par une crue printanière de grande ampleur (de mai à juillet) et qui représente environ $40 \%$ du volume annuel d'écoulement. La crue est suivie d'un étiage estival correspondant au débit moyen annuel, alors que la crue automnale, de faible ampleur, est suivie d'un étiage d'hiver très prononcé (Hydrotech Inc., 1988, 1991 ; Lupien, Rosenberg et Associés, 1991). Les débits moyens annuels des trois rivières présentent par ailleurs des écarts importants (tabl. I). Le débit moyen de la rivière Nottaway est de l'ordre de $1104,5 \mathrm{~m}^{3} / \mathrm{s}$, soit plus du triple de celui de la rivière Broadback $\left(360,5 \mathrm{~m}^{3} / \mathrm{s}\right)$ et nettement supérieur à celui de la rivière de Rupert $\left(845,7 \mathrm{~m}^{3} / \mathrm{s}\right)$. II en est de même pour les débits minimal et maximal moyens annuels. Pour chacune des rivières, ils passent du simple au double.

\section{ÉROSION DES BERGES}

\section{FACTEURS RESPONSABLES DE L'ÉROSION}

Plusieurs facteurs responsables de l'érosion riveraine doivent être considérés : la morphologie et la composition des berges, l'efficacité des agents d'érosion dont les vagues, les courants, les glaces et l'ensemble des autres 
TABLEAU I

Débits moyens annuels et bathymétrie des rivières étudiées

\begin{tabular}{lccc}
\hline & Nottaway & Broadback & de Rupert \\
\hline Débit moyen $\left(\mathrm{m}^{3} / \mathrm{s}\right)$ & 1104,5 & 360,5 & 845,7 \\
Débit minimum $\left(\mathrm{m}^{3} / \mathrm{s}\right)$ & 850,0 & 185,0 & 586,9 \\
Débit maximum $\left(\mathrm{m}^{3} / \mathrm{s}\right)$ & 1631,3 & 421,7 & 1099,8 \\
Profondeur moyenne $(\mathrm{m})$ & 4,3 & 4,3 & 5,5 \\
Largeur moyenne $(\mathrm{m})$ & 870 & 440 & 390 \\
\hline
\end{tabular}

Source : Hydrotech Inc. $(1988,1991)$.

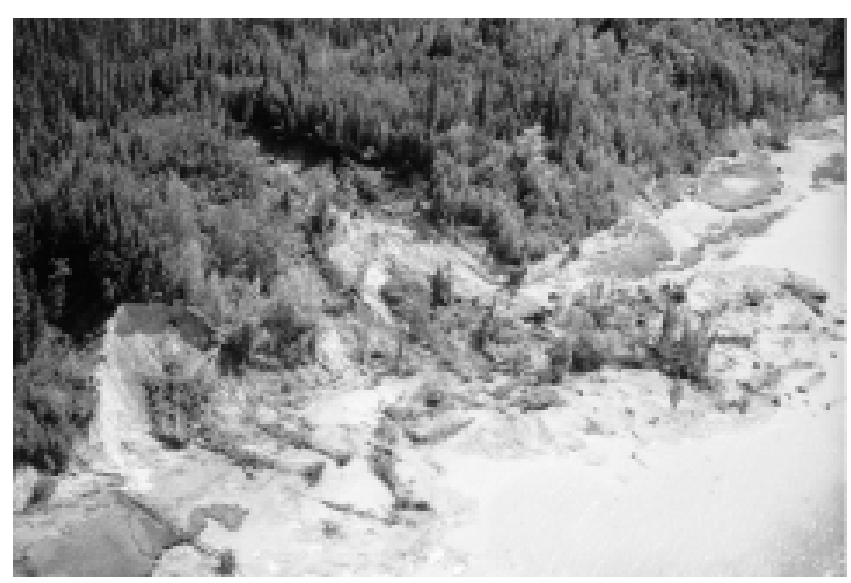

FIGURE 2. Accumulation de débris au pied de la berge résultant de glissements de terrain (rive droite de la rivière Broadback).

Mound debris on the river bank resulting from landslides (right bank of Rivière Broadback).

agents qui affectent la résistance des matériaux de la berge, ainsi que les cycles de gel-dégel ou de mouillage-séchage, la dessiccation par le vent et le soleil, le ruissellement des eaux de surface et la modification des écoulements souterrains (Sylvester, 1974 ; Thorne, 1982 ; Lawson, 1985 ; Knighton, 1998). L'infiltration de l'eau de surface dans les sédiments peut également diminuer la résistance des matériaux (Carson et Lavoie, 1981 ; Thorne, 1982 ; Gostelow, 1996). Les dépôts argileux sont particulièrement sensibles aux variations hydriques internes, en particulier si ce sont des argiles altérées in situ par la perte de leur teneur en sel, comme c'est le cas des argiles marines de la côte est de la baie de James (Carson, 1981 ; SEBJ, 1984).

Les diverses études réalisées le long des rivières de la baie de James (Sogeam, 1980a ; Carter, 1989 ; Bégin et Filion, 1987 ; Sogeam-Tecsult Inc., 1992, 1993 ; Lupien, Rosenberg et Associés, 1991, 1992 ; Hydrotech Inc., 1988, 1991) ont démontré que l'érosion s'effectue principalement au niveau du plan d'eau, au pied des berges, et en partie dans le lit de la rivière. Les berges exposées aux vagues et aux courants sont plus susceptibles d'être érodées, en période de crues, lors de fluctuations des niveaux d'eau ou par forts vents (Sogeam, 1980a; Hydrotech Inc., 1988, 1991 ; Carter, 1989 ; Lupien, Rosenberg et Associés, 1991, 1992). C'est d'ailleurs lors des crues printanières que les charges sédimentaires des rivières sont les plus élevées
(Hydrotech Inc., 1988, 1991). Les observations tendent également à montrer que l'érosion riveraine touche surtout les berges argileuses ou sableuses dont les matériaux sont facilement érodables (Sogeam-Tecsult Inc., 1992, 1993 ; Lupien, Rosenberg et Associés,1991). L'exposition de la berge aux vents dominants contribue à augmenter considérablement son érosion, en particulier dans les zones où la largeur du plan d'eau est suffisante pour engendrer de fortes vagues. On sait que l'énergie des vagues dépend dans ce cas de la vitesse et de la course du vent, et de la largeur du plan d'eau (fetch) ou de la surface d'eau libre sur laquelle agit le vent (Lawson, 1985). L'attaque des vagues est d'autant plus efficace que la berge est sans protection (ni plage ni végétation).

L'action des glaces contribue aussi à l'érosion des berges (Sylvester, 1974 ; Lawson, 1985). Si un couvert de glace continu a pour effet de protéger les berges contre les vagues et les courants, un couvert de glace morcelé peut au contraire provoquer l'érosion des berges. Au dégel, le couvert de glace se fragmente, formant des radeaux de glace qui dérivent avec le courant de la rivière. Ces glaces peuvent saper et éroder les berges. L'érosion s'aggrave aussi lorsque le couvert de glace, soulevé par la montée rapide des eaux, arrache de toute sa masse le matériel aux parois des talus (Dionne, 1976, 1978 ; Laverdière et Guimont, 1975, 1979, 1980 ; Guimont et Laverdière, 1983). Dans les rivières nordiques, des ponts suspendus de frasil sous les glaces réduisent les sections mouillées et entraînent l'érosion du lit. Au printemps, le couvert de glace, en se soulevant, arrache et transporte des tonnes de sédiments auxquels il s'est soudé au cours de l'hiver. De même, le raclage et le décapage du lit fluvial et des berges, par les blocs et les cailloux fixés à la banquise fluviale, ou poussés par les glaces en mouvement ou par les radeaux glaciels entraînés par le courant, contribuent au nettoyage des matériaux provenant des talus riverains, à l'enfoncement du lit fluvial et au recul des versants.

\section{CYCLES D'ÉROSION}

Les berges constituées de matériaux argileux ou limonoargileux sont sensibles à l'érosion riveraine, en particulier si elles forment de hautes terrasses escarpées. Pour ce type de berges, le processus d'érosion se déroule très souvent de façon cyclique (Mollard et Janes, 1985 ; Beven et Carling, 1989 ; Gostelow, 1996). Dans un premier temps, l'action répétée des vagues au pied du talus enlève des matériaux et affaiblit la stabilité de la pente jusqu'à la rupture (Lupien, Rosenberg et Associés, 1991). Lorsque le point de rupture est atteint, s'engage alors un mouvement de masse sous la forme d'un glissement, d'un éboulement ou d'un décrochement d'ampleur variable suivant les conditions locales. Dans le cas d'un glissement, la rupture s'étend habituellement le long d'un plan de cisaillement basal à peu près parallèle à la surface du sol (Mollard et Janes, 1985 ; Beven et Carling, 1989 ; Gostelow, 1996). Les matériaux qui glissent le long de la pente viennent alors s'échouer sur la rive (fig. 2), formant momentanément un rempart contre les vagues et les courants. Ces matériaux sont ensuite érodés et évacués par les eaux de la rivière dès la première année ou 
FIGURE 3. Schéma illustrant les principales phases d'évolution d'une terrasse argileuse (modifié de SEBJ, 1991).

Schematic graph showing the mechanism of clay river bank evolution at various stages (modified SEBJ, 1991).

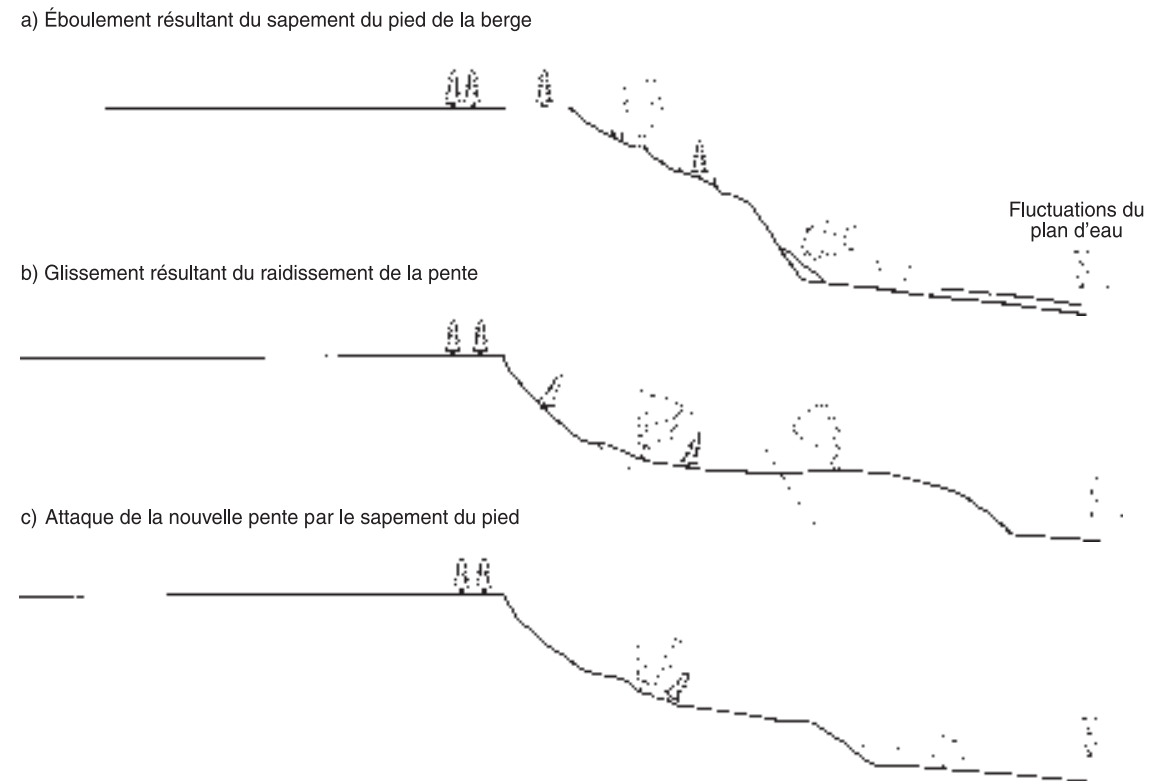

TABLEAU II

Répartition des berges selon la nature des matériaux pour les rivières Nottaway, Broadback et de Rupert (tronçon aval)

\begin{tabular}{|c|c|c|c|c|c|}
\hline \multirow[b]{2}{*}{$\begin{array}{l}\text { Composition dominante de la } \\
\text { berge }\end{array}$} & \multirow[b]{2}{*}{ totale } & \multicolumn{2}{|c|}{ Longueur des berges $(\mathrm{km})$} & \multirow[b]{2}{*}{$\begin{array}{l}\text { Actives } \\
(\%)\end{array}$} & \multirow[b]{2}{*}{$\begin{array}{c}\% \text { de berge active selon la nature } \\
\text { des matériaux }\end{array}$} \\
\hline & & $\begin{array}{l}\text { Totale } \\
(\%)\end{array}$ & actives & & \\
\hline Argile & $591,0^{*}$ & $(51,2 \%)$ & $93,7^{*}$ & $(15,9 \%)^{\star \star}$ & 83,1 \\
\hline Blocs et cailloux & 178,6 & $(15,5 \%)$ & 7,7 & $(4,3 \%)$ & 6,8 \\
\hline Roc & 162,4 & $(1,1 \%)$ & 3,6 & $(2,2 \%)$ & 3,3 \\
\hline Tourbière & 84,1 & $(7,3 \%)$ & 2,3 & $(2,7 \%)$ & 2,0 \\
\hline Till & 60,9 & $(5,3 \%)$ & 0,5 & $(0,8 \%)$ & 0,4 \\
\hline Sable & 42,0 & $(3,6 \%)$ & $<0,1$ & $(<0,2 \%)$ & $<0,1$ \\
\hline Till argileux & 18,0 & $(1,6 \%)$ & 0,2 & $(1,1 \%)$ & 0,2 \\
\hline Till sur roc & 12,2 & $(1,1 \%)$ & 3,8 & $(31,1 \%)$ & 3,4 \\
\hline Autres & 4,1 & $(<0,1 \%)$ & 0,8 & $(19,5 \%)$ & 0,7 \\
\hline Total des berges & 1153,3 & $(100 \%)$ & 112,7 & $(9,8 \%)$ & $100 \%$ \\
\hline
\end{tabular}

* Les valeurs représentent les longueurs des deux rives et des îles ;

${ }^{* *}$ Pourcentages obtenus à partir des berges actives/berges totales.

Source : Sogeam-Tecsult Inc. (1992).

l'année suivante selon l'importance de l'accumulation. On reconnaît les glissements récents par la présence de ces loupes de glissement au pied de la berge. Ces accumulations se composent le plus souvent d'un mélange de tourbe, d'argile ou d'autres matériaux (sable, gravier), auxquels s'entremêlent des troncs et des branches d'arbres. Un fois les débris délogés, laissant ainsi la berge à nouveau sans protection, on retrouve alors des conditions semblables à celles de l'état initial, et le cycle d'érosion peut recommencer. La figure 3 en illustre les principales phases.

\section{COMPOSITION DES BERGES ET FORMES D'ÉROSION}

\section{CARACTÉRISTIQUES MORPHO-SÉDIMENTOLOGIQUES}

Les berges des rivières à l'étude sont composées de matériaux qui témoignent des divers contextes géologiques de mise en place. Selon la nature des sédiments et la morphologie des berges, certaines d'entre elles sont plus touchées par l'érosion fluviale que d'autres. Le tableau II fournit la répartition des berges actives selon la nature des matériaux pour l'ensemble des trois rivières et la figure 4 montre la répartition des matériaux pour chacune des rivières dans leur portion aval. Les rivières totalisent seulement $10 \%$ de berges actives, et les berges de nature argileuse apparaissent particulièrement sensibles à l'érosion.

Les caractéristiques morpho-sédimentologiques des berges actives pour chacune des rivières (tabl. III, IV et V) révèlent que les berges de nature argileuse avec des pentes hautes et abruptes sont les plus abondantes. Dans le cas de la rivière Nottaway, de son embouchure jusqu'en aval de la rivière Soscumica, l'érosion atteint plus de $13 \mathrm{~km}$ de berges constituées uniquement d'argile et plus de $9 \mathrm{~km}$ de berges 

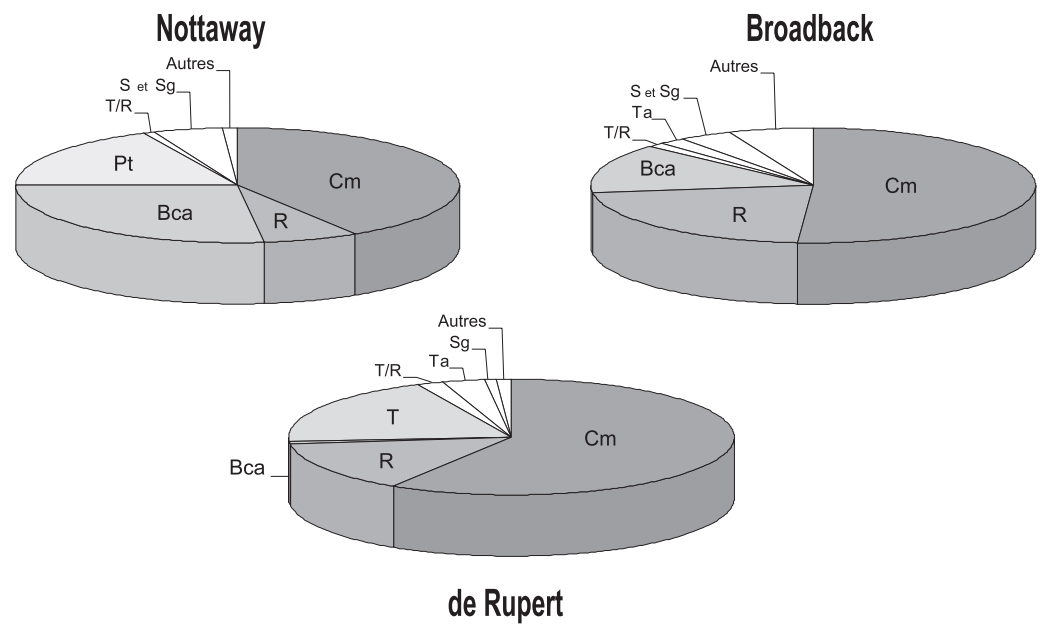

TABLEAU III

Caractéristiques morpho-sédimentologiques des berges actives $d u$ tronçon aval de la rivière Nottaway

\begin{tabular}{|c|c|c|c|}
\hline Composition de la berge & $\begin{array}{l}\text { Hauteur du } \\
\text { talus }(\mathrm{m})\end{array}$ & $\begin{array}{l}\text { Inclinaison de } \\
\text { la pente }\left(^{\circ}\right)\end{array}$ & $\begin{array}{l}\text { Longueur de } \\
\text { la berge }(\mathrm{km})\end{array}$ \\
\hline Argile & $<2$ & $>25^{\circ}$ & 0,3 \\
\hline Argile & $2-10$ & $5^{\circ}-14^{\circ}$ & 0,7 \\
\hline Argile & $2-10$ & $14^{\circ}-25^{\circ}$ & 2,8 \\
\hline Argile & $2-10$ & $>25^{\circ}$ & 3,9 \\
\hline Argile & $10-20$ & $14^{\circ}-25^{\circ}$ & 0,3 \\
\hline Argile & $10-20$ & $>25^{\circ}$ & 5,1 \\
\hline Blocs/cailloux sur argile & $<2$ & $5^{\circ}-14^{\circ}$ & 0,5 \\
\hline Blocs/cailloux sur argile & $<2$ & $14^{\circ}-25^{\circ}$ & 0,3 \\
\hline Blocs/cailloux sur argile & $2-10$ & $5^{\circ}-14^{\circ}$ & 1,1 \\
\hline Blocs/cailloux sur argile & $2-10$ & $14^{\circ}-25^{\circ}$ & 3,1 \\
\hline Blocs/cailloux sur argile & $2-10$ & $>25^{\circ}$ & 0,5 \\
\hline Blocs/cailloux sur argile & $10-20$ & $14^{\circ}-25^{\circ}$ & 0,4 \\
\hline Blocs/cailloux sur argile & $10-20$ & $>25^{\circ}$ & 1,2 \\
\hline Blocs/cailloux sur argile & $>20$ & $14^{\circ}-25^{\circ}$ & 1,1 \\
\hline Sable sur argile & $2-10$ & $>25^{\circ}$ & 0,5 \\
\hline Sable sur argile & $10-20$ & $>25^{\circ}$ & 2,0 \\
\hline Sable sur argile & $>20$ & $>25^{\circ}$ & 1,2 \\
\hline Sable grossier sur argile & $2-10$ & $14^{\circ}-25^{\circ}$ & 0,2 \\
\hline Tourbière sur argile & $<2$ & $>25^{\circ}$ & 0,9 \\
\hline Tourbière sur argile & $2-10$ & $14^{\circ}-25^{\circ}$ & 0,4 \\
\hline Tourbière sur argile & $2-10$ & $>25^{\circ}$ & 7,0 \\
\hline Tourbière sur argile & $10-20$ & $>25^{\circ}$ & 1,2 \\
\hline Blocs sur argile & $2-10$ & $5^{\circ}-14^{\circ}$ & 2,9 \\
\hline Blocs sur argile & $2-10$ & $>25^{\circ}$ & 3,4 \\
\hline Till sur roc & $10-20$ & $<5^{\circ}$ & 0,6 \\
\hline Blocs & $2-10$ & $14^{\circ}-25^{\circ}$ & 1,6 \\
\hline Autres* & & & 9,6 \\
\hline \multicolumn{3}{|c|}{ Total des berges actives du tronçon aval } & 55,7 \\
\hline \multicolumn{3}{|c|}{ Total des berges du tronçon aval } & 370,3 \\
\hline \multicolumn{3}{|c|}{ Total des berges actives de l'ensemble de la rivière } & 163,5 \\
\hline \multicolumn{3}{|c|}{ Total des berges de l'ensemble de la rivière } & 2999,0 \\
\hline
\end{tabular}

*Autres comprend les blocs et cailloux (5,9\%), le roc (3,6\%) et le sable $(<0,1)$.

Source : Sogeam-Tecsult Inc. (1992, 1993).
FIGURE 4. Diagrammes circulaires montrant la composition des berges actuelles pour chacune des rivières étudiées (tronçon aval). Légende: Bca : blocs et cailloux; $\mathrm{Cm}$ : argile ; $\mathrm{Pt}$ : tourbe ; $\mathrm{S}$ : sable $; \mathrm{Sg}$ : sable et gravier ; Ta ; till argileux; T/R till sur roc ; $\mathrm{R}$ : roc.

Circular diagrams showing the material composition of the actual river banks for each of the studied rivers (downstream). Legend: Bca: blocks and pebbles; Cm: clay; Pt: peat; S: sand; Sg: sand and gravels; Ta: clayey till; T/R: till on rock; $R$ : rock. argileuses coiffées de tourbe (tabl. III). II faut également y ajouter $18 \mathrm{~km}$ de berges argileuses montrant des accumulations de blocs et cailloux ou de sable sur les plages. Ces matériaux proviennent du transport par les glaces ou par les courants. Pour la rivière Broadback, en aval du lac Giffard, plus de $5 \mathrm{~km}$ de berges argileuses sont actives (tabl. IV), et pour la rivière de Rupert, en aval du lac Némiscau, on compte plus de $40 \mathrm{~km}$ de berges argileuses actives, en particulier sur les talus abrupts de plus de $25^{\circ}$ (tabl. V). En comparant la longueur des berges actives dans la portion aval des trois rivières (tabl. III, IV et V), on constate par ailleurs que les rivières Nottaway et de Rupert comptent sensiblement la même longueur de berges actives, soit $55 \mathrm{~km}$ $(13,3 \%)$ et $51 \mathrm{~km}(11,0 \%)$ respectivement, alors que la rivière Broadback compte seulement $6 \mathrm{~km}(1,9 \%)$ de berges actives (Sogeam-Tecsult Inc., 1992, 1993).

Si l'on tient compte de la longueur totale de chacune des rivières (tabl. III, IV et V), il ressort que les berges de la rivière Nottaway sont plus souvent érodées que celles des autres rivières. On y dénombre en effet $163 \mathrm{~km}(5,4 \%)$ de berges actives, et seulement $64 \mathrm{~km} \mathrm{(2,7 \% )} \mathrm{et} 80 \mathrm{~km}(2,2 \%)$ pour les rivières Broadback et de Rupert. La rivière Nottaway traverse des terrains argileux sur plusieurs dizaines de kilomètres, depuis les lacs Waswanipi et Soscumica jusqu'à son embouchure. Dans le secteur SoscumicaWaswanipi, les terrains sont surtout constitués d'argile glacio-lacustre, tandis qu'en aval dominent des argiles marines. La rivière recoupe aussi des matériaux glaciaires (till de Cochrane), notamment au nord du lac Soscumica (Poly-Géo Inc., 1991).

La répartition des berges actives de la rivière Broadback varie beaucoup d'une section à l'autre. Elles sont moins nombreuses en amont, où dominent les tills et les affleurements rocheux, qu'en aval, où se situent les plaines d'argile marine (Sogeam-Tecsult Inc., 1992, 1993). Pour la rivière de Rupert, les berges en activité occupent surtout la portion aval du cours d'eau près de son embouchure, où dominent de hautes terrasses d'argile marine. On dénombre un peu plus de $40 \mathrm{~km}$ de berges vives le long de ce parcours. Les cicatrices de glissements de terrain et d'anciennes coulées y sont nombreuses (Sogeam-Tecsult Inc., 1992). 
TABLEAU IV

Caractéristiques morpho-sédimentologiques des berges actives du tronçon aval de la rivière Broadback

\begin{tabular}{|c|c|c|c|}
\hline $\begin{array}{l}\text { Composition de } \\
\text { la berge }\end{array}$ & $\begin{array}{l}\text { Hauteur du } \\
\text { talus }(\mathrm{m})\end{array}$ & $\begin{array}{c}\text { Inclinaison de la } \\
\text { pente }\left(^{\circ}\right)\end{array}$ & $\begin{array}{l}\text { Longueur de la } \\
\text { berge }(\mathrm{km})\end{array}$ \\
\hline Argile & $<2$ & $<5^{\circ}$ & $<0,1$ \\
\hline Argile & $2-10$ & $14^{\circ}-25^{\circ}$ & 0,3 \\
\hline Argile & $2-10$ & $>25^{\circ}$ & 1,7 \\
\hline Argile & $10-20$ & $14^{\circ}-25^{\circ}$ & 0,2 \\
\hline Argile & $10-20$ & $>25^{\circ}$ & 1,7 \\
\hline Argile & $>20$ & $>25^{\circ}$ & 1,3 \\
\hline Till & $2-10$ & $>25^{\circ}$ & 0,2 \\
\hline Till & $10-20$ & $>25^{\circ}$ & 0,3 \\
\hline Till argileux & $2-10$ & $>25^{\circ}$ & 0,2 \\
\hline \multicolumn{3}{|c|}{ Total des berges actives du tronçon aval } & 6,0 \\
\hline \multicolumn{3}{|c|}{ Total des berges du tronçon aval } & 318,7 \\
\hline \multicolumn{3}{|c|}{$\begin{array}{l}\text { Total des berges actives de l'ensemble de la } \\
\text { rivière }\end{array}$} & 64,3 \\
\hline \multicolumn{3}{|c|}{ Total des berges de l'ensemble de la rivière } & 2367,0 \\
\hline
\end{tabular}

Source : Sogeam-Tecsult Inc. (1992, 1993).

\section{PHÉNOMÈNES ET FORMES D'ÉROSION}

Les phénomènes d'érosion observés le long des rivières Nottaway, Broadback et de Rupert dépendent largement des caractéristiques morpho-sédimentologiques des berges. Ils sont fréquents le long des pentes abruptes et argileuses (fig. 5 et 6). Les glissements sont habituellement associés à des mouvements rapides, alors que les éboulements sont des mouvements plus lents qui résultent de l'érosion du pied des talus par les vagues et les courants. À mesure que progresse l'entaille basale, les matériaux se détachent par tranches successives dépassant rarement un mètre d'épaisseur (Lupien, Rosenberg et Associés, 1991, p. 19). Aux endroits dépourvus de végétation, les terrasses d'argile sont le plus souvent ravinées par les eaux de ruissellement. Le sapement latéral des eaux fluviales cause également des décrochements et des éboulements dans les terrasses sableuses ou sablo-graveleuses, dont les matériaux sont peu cohésifs. Dans les dépôts de till, on observe plutôt de petits effondrements causés par l'enlèvement des blocs et cailloux prélevés soit par les glaces flottantes ou délogés par l'action des vagues et des courants. Parfois, la liquéfaction des matériaux fins entraîne des mouvements localisés

Dans les hautes falaises d'argile, les glissements sont plus nombreux et parfois spectaculaires. Certains d'entre eux atteignent en effet plusieurs mètres de hauteur (15 à $20 \mathrm{~m}$ ) et plusieurs dizaines de mètres de largeur. Ils entaillent profondément les terrasses en y formant des cuvettes ou des amphithéâtres. Ces derniers prennent généralement la forme d'un goulet ou d'un entonnoir en bordure des terrasses (fig. 7). À l'intérieur des amphithéâtres de
TABLEAU V

Caractéristiques morpho-sédimentologiques des berges actives du tronçon aval de la rivière de Rupert

\begin{tabular}{|c|c|c|c|}
\hline $\begin{array}{l}\text { Composition de } \\
\text { la berge }\end{array}$ & $\begin{array}{l}\text { Hauteur du } \\
\text { talus }(\mathrm{m})\end{array}$ & $\begin{array}{c}\text { Inclinaison de la } \\
\text { pente }\left(^{\circ}\right)\end{array}$ & $\begin{array}{c}\text { Longueur de la } \\
\text { berge }(\mathrm{km})\end{array}$ \\
\hline Argile & $<2$ & $5^{\circ}-14^{\circ}$ & 0,1 \\
\hline Argile & $<2$ & $>25^{\circ}$ & 0,2 \\
\hline Argile & $2-10$ & $>25^{\circ}$ & 10,2 \\
\hline Argile & $10-20$ & $>25^{\circ}$ & 2,6 \\
\hline Argile & $>20$ & $>25^{\circ}$ & 31,4 \\
\hline $\begin{array}{l}\text { Blocs et cailloux } \\
\text { sur argile }\end{array}$ & $2-10$ & $5^{\circ}-14^{\circ}$ & 0,2 \\
\hline Blocs et cailloux & $<2$ & $5^{\circ}-14^{\circ}$ & $<0,1$ \\
\hline $\begin{array}{l}\text { Tourbière sur } \\
\text { argile }\end{array}$ & $2-10$ & $>25^{\circ}$ & 2,6 \\
\hline $\begin{array}{l}\text { Sable grossier } \\
\text { sur argile }\end{array}$ & $<2$ & $5^{\circ}-14^{\circ}$ & 0,1 \\
\hline Till sur roc & $10-20$ & $>25^{\circ}$ & 1,2 \\
\hline Tourbière & $<2$ & $>25^{\circ}$ & 2,3 \\
\hline \multicolumn{3}{|c|}{ Total berges actives du tronçon aval } & 51,0 \\
\hline \multicolumn{3}{|c|}{ Total des berges du tronçon aval } & 465,3 \\
\hline \multicolumn{3}{|c|}{ Total berges actives de l'ensemble de la rivière } & 79,9 \\
\hline \multicolumn{3}{|c|}{ Total des berges de l'ensemble de la rivière } & 3631,0 \\
\hline
\end{tabular}

Source : Sogeam-Tecsult Inc. (1992, 1993).

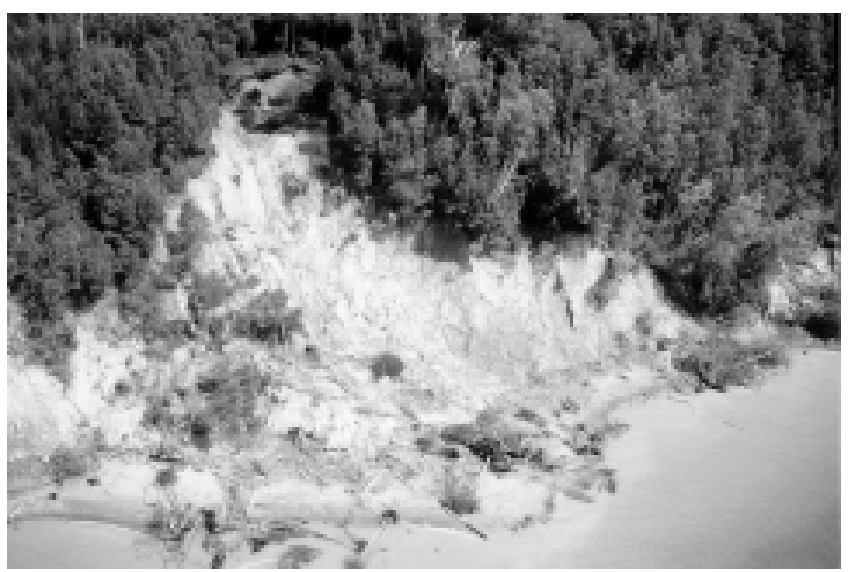

FIGURE 5. Glissement dans une terrasse argileuse entraînant la chute des arbres (rive droite de la rivière Broadback).

Landslide in a clay terrace causing trees to fall along the slope (right bank of Rivière Broadback ).

glissement, les surfaces nouvellement effondrées, bouleversées et dénudées, apparaissent en replats étagés (fig. 7). Généralement, le glissement de matériaux argileux a provoqué le déracinement de nombreux arbres qui sont venus s'échouer au pied de la berge. Ces bourrelets de matériaux protègent momentanément la berge contre l'assaut des vagues et servent en quelque sorte de rempart au pied des talus. Les anciens glissements et les cicatrices de coulées sont relativement nombreux à l'embouchure de la rivière de Rupert (Sogeam, 1980a; Sogeam-Tecsult Inc., 1992). La 


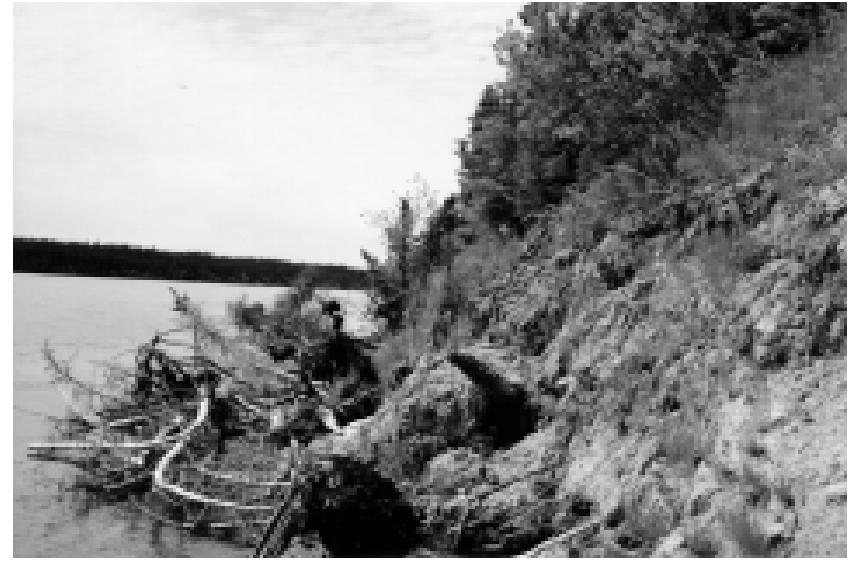

FIGURE 6. Sapement à la base d'une terrasse argileuse provoquant la chute de matériaux le long de la pente.

Clay terrace affected by mass movement (crumbling) related to basal erosion.

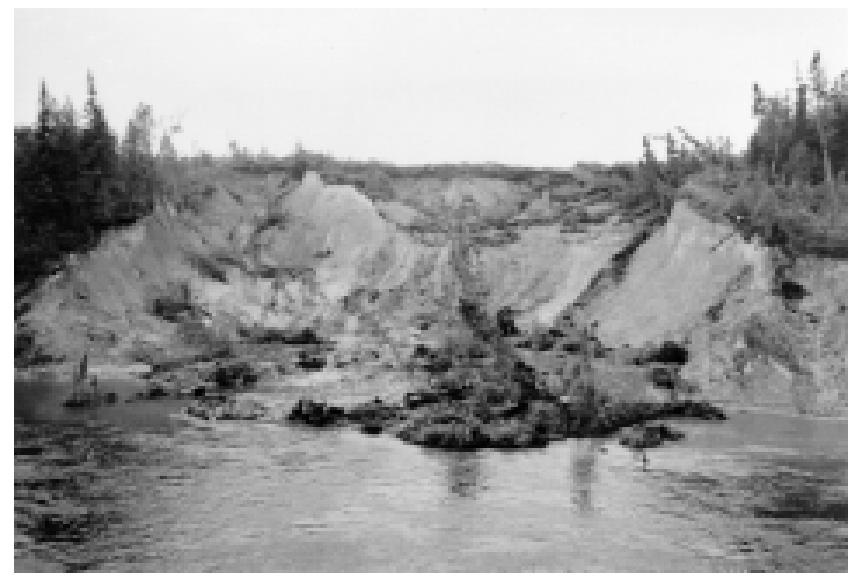

FIGURE 7. Glissement de terrain dans une terrasse argileuse près de l'embouchure de la rivière de Rupert (rive gauche $\mathrm{km} 7$ ).

Landslide in a clay terrace located near the mouth of Rivière de Rupert (left bank at km 7).

contiguïté de certains glissements et coulées d'argile ne laisse en bordure des terrasses qu'une série d'éperons résiduels qui confèrent à la berge un tracé dentelé (fig. 8). On trouve également ces formes le long des rivières Nottaway et Broadback, mais en moins grand nombre.

Les coulées argileuses sont dues à un mouvement rétrogressif qui produit une dépression dont la superficie est grande par rapport à la profondeur du plan de rupture (Lupien, Rosenberg et Associés, 1991, p. 21). Les argiles marines de la côte jamésienne sont des matériaux particulièrement vulnérables à de tels mouvements de masse (Ballivy et al., 1975 ; SEBJ,1984). Ces phénomènes se développent pratiquement toujours dans des matériaux argileux dont le Mhum $\mathrm{H} / \mathrm{c}_{\mathrm{u}}{ }^{1}$ est de l'ordre de 6,0 à 6,5 (SEBJ, 1984). De tel-

1. Mhum = masse volumique humide; $\mathrm{H}=$ profondeur; $\mathrm{c}_{\mathrm{u}}=$ résistance au cisaillement non drainé.

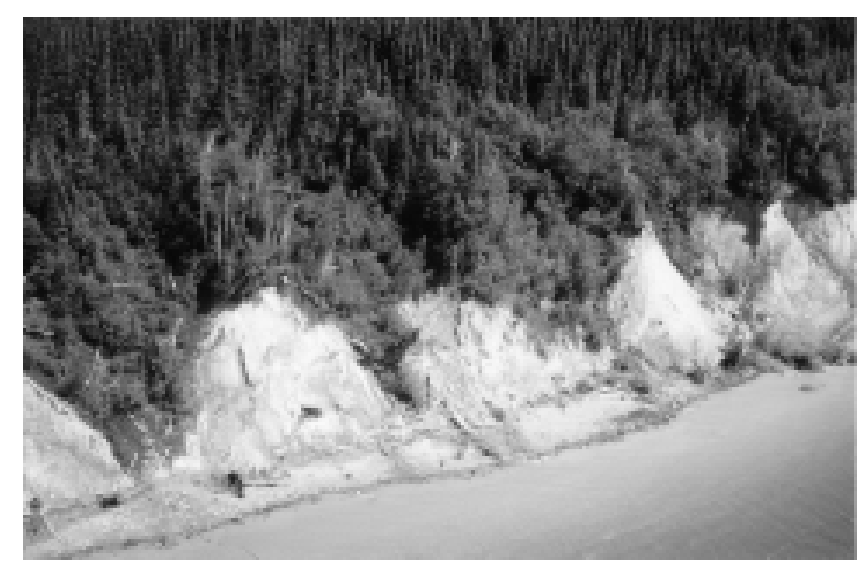

FIGURE 8. Terrasse argileuse le long de la rivière Broadback. Des glissements rapprochés découpent la terrasse en éperons trapézoïdaux.

Close in space landslides shape the river bank in the form of trapezoidal spurs.

les valeurs confèrent aux matériaux une très faible résistance au cisaillement. Cette faible résistance combinée à la . hauteur élevée des terrasses (parfois plus de $20 \mathrm{~m}$ ) favorisent les mouvements de masse. On comprend qu'ils soient nombreux à l'embouchure de la rivière de Rupert où dominent les hautes terrasses d'argile ; des cicatrices de glissement y sont particulièrement abondantes. Cette situation s'apparente d'ailleurs à celle de la Grande Rivière qui se caractérise également par la présence de très hautes terrasses argileuses qui ont été atteintes par de nombreux mouvements de masse (Lupien, Rosenberg et Associés, 1991 ; SEBJ, 1991).

\section{CHARGES SÉDIMENTAIRES ET TAUX D’ÉROSION}

\section{BILAN DES CHARGES SÉDIMENTAIRES}

Les charges sédimentaires transportées par les rivières Nottaway, Broadback et de Rupert vers la baie de Rupert sont très variables d'une rivière à l'autre. La rivière Nottaway fournit $59 \%$ de la charge totale annuelle de sédiments, tandis que les rivières Broadback et de Rupert contribuent pour leur part respective à $14 \%$ et $27 \%$ du bilan sédimentaire (tabl. VI). Ces apports proviennent de l'érosion soit du lit des rivières, soit des berges ou des affluents qui se jettent dans ces rivières. Pour les berges seulement, les charges solides sont de l'ordre de 230,0 t/a pour la rivière Nottaway, 68,5 t/a pour la Broadback et 112,9 t/a pour la rivière de Rupert (tabl. VII) ; ce qui représente en moyenne 1314,3 t/km/an de sédiments, 548,1 et 705,9 t/km/an respectivement par kilomètre de rive pour chaque rivière (Hydrotech Inc., 1991). Enfin, selon le calcul des charges sédimentaires, les portions aval des rivières contribuent en moyenne à $50 \%$ des apports totaux de sédiments déversés dans la baie de Rupert et ce pourcentage atteint environ $70 \%$ en période de crues, ce qui témoigne d'une activité accrue de l'érosion de leurs berges durant cette période (Hydrotech Inc., 1977, 1991). 
TABLEAU VI

Bilans sédimentaires moyens des vivières Nottaway, Broadback et de Rupert, vers la baie de Rupert (suspension et charriage)*

\begin{tabular}{lcccc}
\hline & Suspension (t/a) & Charriage (t/a) & Total (t/a) & $\%$ \\
\hline Nottaway & 537,217 & 53,600 & 590,817 & 59 \\
Broadback & 128,333 & 16,450 & 144,783 & 14 \\
De Rupert & 217,075 & 48,700 & 265,775 & 27 \\
Baie de Rupert & 882,625 & 118,750 & 1001,375 & 100 \\
\hline
\end{tabular}

*Les bilans sédimentaires ont été calculés à partir de l'analyse des concentrations de matières prélevées dans les échantillons d'eau, lors de campagnes de terrain des années 1976, 1977 et 1979. Ces données de base ont servi au développement de modèles de simulation en conditions naturelles et après aménagement des rivières du complexe NBR (Hydrotech Inc., 1977, 1991). Les données de débit ont été fournies et mises à jour par le Service hydraulique d'Hydro-Québec à partir des stations de jaugeage du ministère de l'Environnement. Elles sont basées sur un échantillonnage d'une durée de 47 ans (1935-1982).

Source : Hydrotech Inc. (1991).

\section{TABLEAU VII}

Contribution spécifique des berges en sédiments (suspension et charriage) des rivières Nottaway, Broadback et de Rupert vers la baie de Rupert - tronçon aval

\begin{tabular}{lcc}
\hline Contribution des berges & $\begin{array}{c}\text { Débits solides } \\
(\mathrm{t} / \mathrm{a})\end{array}$ & $\begin{array}{c}\text { Dégradation spécifique } \\
(\mathrm{t} / \mathrm{km} / \mathrm{a})\end{array}$ \\
\hline Nottaway $(175 \mathrm{~km})$ & 230010 & $1314,3^{*}$ \\
Broadback $(125 \mathrm{~km})$ & 68514 & 548,1 \\
De Rupert $(160 \mathrm{~km})$ & 112951 & 705,9 \\
\hline
\end{tabular}

*Cette valeur est obtenue en divisant 230010 par $175 \mathrm{~km}$. Source : Hydrotech Inc. (1991).

\section{RECUL DES RIVES ET TAUX D'ÉROSION}

Le taux d'érosion des berges (Hydrotech Inc., 1988, 1991 ; Lupien, Rosenberg et Associés, 1991) est évalué à partir du volume de matériaux déversés dans le cours d'eau. Ainsi, pour le tronçon aval de la rivière Broadback, on obtient un taux d'érosion unitaire d'environ $0,1 \mathrm{~m}^{3}$ par mètre linéaire de rive, en considérant un volume moyen de $2 \mathrm{t} / \mathrm{m}^{2}$ par année (tabl. VII), soit une érosion moyenne de l'ordre de $2 \mathrm{~cm} /$ an pour une hauteur de berge de $5 \mathrm{~m}$ (Hydrotech Inc., 1991). Ce taux correspond à celui calculé par Lupien, Rosenberg et Associés (1991) pour la même rivière. Bien que les taux de recul des berges ne soient par fournis pour les rivières Nottaway et de Rupert, on peut s'attendre à des taux d'érosion comparables ou supérieurs à celui calculé pour la rivière Broadback. Les valeurs obtenues pour les charges solides (tabl. VII) indiquent en effet que la dégradation des berges est en moyenne plus élevée pour les deux autres rivières que pour la rivière Broadback (Hydrotech Inc., 1991).

Enfin, en comparant les taux d'érosion des berges de la rivière Broadback avec celui de la Grande Rivière (LG1) dans leurs tronçons aval, on constate des différences très marquées qui s'expliquent par l'hydrologie et la géomorphologie. La Grande Rivière présente en effet des débits beaucoup plus élevés (soit entre 2500 et $3000 \mathrm{~m}^{3} / \mathrm{s}$ ) que ceux de la rivière Broadback et surtout, elle est constituée de très hautes terrasses d'argile montrant de nombreux glissements et coulées d'argile (SEBJ, 1991). Le taux d'érosion des berges de la portion aval de la Grande Rivière est estimé entre 0,35 et $0,50 \mathrm{~m} / \mathrm{an}$, soit vingt cinq fois plus que celui de la rivière Broadback (SEBJ, 1991). Le volume de matériaux livrés à cette dernière est en effet évalué à $16200 \mathrm{~m}^{3} / \mathrm{an}$, alors que celui de la Grande Rivière en conditions naturelles est de l'ordre de $125000 \mathrm{~m}^{3}$ /an (SEBJ, 1991 ; Lupien, Rosenberg et Associés, 1991). Bien que les berges de la portion aval de la rivière Broadback soient constituées principalement de matériaux argileux, les mouvements de masse y sont relativement moins fréquents en raison de la plus faible proportion de talus élevés. On estime que les glissements fournissent moins de $1700 \mathrm{~m}^{3}$ de matériaux en moyenne à la rivière Broadback (Lupien, Rosenberg et Associés, 1991). Pour la Grande Rivière, le nombre moyen de glissements par année est évalué à huit, pour un volume de matériaux annuel moyen de l'ordre de $66700 \mathrm{~m}^{3}$, dont environ $25000 \mathrm{~m}^{3}$ proviendraient de l'aval (SEBJ, 1991).

Les sédiments transportés vers la baie de Rupert témoignent néanmoins de l'importance de l'érosion fluviale des rivières Nottaway, Broadback et de Rupert et de leurs affluents. Cette érosion se traduit par un recul progressif des berges modifiant ainsi le profil des versants et le tracé des rivières. Les escarpements argileux sont les plus sollicités par l'érosion, en particulier en périodes de crues où on enregistre les charges sédimentaires les plus fortes de l'année. Les apports modifient progressivement les conditions sédimentologiques et hydrologiques de la baie de Rupert. On sait que celle-ci est déjà peu profonde, soit 3 à $6 \mathrm{~m}$ en moyenne (SEBJ, 1978 ; Sogeam, 1980b), et que ces apports contribuent à son ensablement et à son comblement, en particulier dans les zones à faible écoulement. Aussi, ces changements modifient peu à peu le patron de circulation des eaux entre la baie de Rupert et la baie de James. S'ajoute la progression des estrans et des hauts fonds qui s'expliquerait par un relèvement isostatique résiduel dont le taux est évalué entre 0,7 et $1,5 \mathrm{~cm}$ par année selon les secteurs (D'anglejan, 1980 ; GEL Inc., 1992). Tous ces éléments contribuent donc à modifier progressivement les conditions de la baie de Rupert et ces changements ont des conséquences directes sur l'ensemble des milieux physique et biologique. 


\section{CONCLUSION}

L'étude des rivières Nottaway, Broadback et de Rupert révèle que les berges en aval sont fortement érodées. La dynamique fluviale met en cause divers agents d'érosion, dont les principaux sont les vagues, les courants et les glaces. Ils agissent surtout à la base des talus, entraînant progressivement la modification des berges et une perte de matériaux le long des pentes. Dans plusieurs cas, l'évolution des berges est liée à un processus cyclique d'érosion qui débute par le sapement des vagues au pied du talus et qui se termine par un mouvement de masse.

Les différents phénomènes et formes d'érosion fluviale observés le long des rivières s'expliquent largement par la nature des matériaux et la géomorphologie des berges actuelles. Les hautes terrasses d'argile sont particulièrement touchées par l'érosion. Les formes d'érosion riveraine les plus spectaculaires se trouvent à l'embouchure de la rivière de Rupert où dominent les hautes falaises argileuses. On peut en effet y observer d'importants glissements de terrain, anciens ou récents, qui confèrent à la rive une morphologie particulière. Les éboulements sont également d'autres formes d'érosion fréquemment observées le long des trois rivières. Les sédiments transportés par les rivières dans la baie de Rupert sont évalués à plus de 1000000 t/a (suspension et charriage) en moyenne. Ils entraînent le comblement progressif de la baie, en particulier dans les zones à faible courant. Cette sédimentation est aggravée par le relèvement isostatique, évalué entre 0,7 et $1,5 \mathrm{~cm} / \mathrm{an}$. Ces modifications transforment les conditions hydrologiques actuelles dans la baie, notamment la circulation des eaux entre la baie de Rupert et la baie de James. Des études plus détaillées des cours d'eau basées sur des modèles hydrodynamiques (transport, charge sédimentaire, vitesse d'écoulement, etc.) permettraient de mieux évaluer les conséquences physiques et biologiques à long terme. Parmi les sujets de recherche qui pourraient compléter et approfondir les travaux antérieurs (Sogeam, 1980b ; D’anglejan, 1980, 1994 ; GEL, 1992) mentionnons l'affaiblissement de l'influence maritime dans l'estuaire, les modifications de l'amplitude de la marée, l'évolution morpho-sédimentologique de la baie de Rupert (rivages, îles, etc.) et leurs conséquences sur la faune et la flore aquatique.

\section{REMERCIEMENTS}

Ces travaux furent réalisés pour le compte de la Société Hydro-Québec. Nous remercions M. Robert Denis (H-Q) pour sa précieuse collaboration. M. Camille Laverdière a aimablement effectué une première lecture du manuscrit, qui fut amélioré grâce aux commentaires de MM. J.-C. Dionne, J.-M. Dubois et d'un lecteur anonyme. M. André Parent du Département de géographie de l'Université du Québec à Montréal s'est chargé de la confection des cartes et figures.

\section{RÉFÉRENCES}

Ballivy, G., Loiselle, A.A. et Pouliot, G., 1975. Quelques caractéristiques géotechniques des dépôts d'argile de la Baie James : les coulées d'argile de Fort Rupert, Québec. Revue canadienne de Géotechnique, 12 : 498510.
Bégin, C. et Filion, L., 1987. Morphologie et interprétation des glissements de terrain de la région de Poste-de-la-Baleine, Québec subarctique. Géographie physique et Quaternaire, 41 : 19-32.

Beven, K. et Carling, P. (édit.), 1989. Floods : Hydrological, Sedimentological and Geomorphological Implications. Wiley, Chichester, 290 p.

Carson, M.A. et Lavoie, G., 1981. Some contraints on the severity of landslide penetration in sensitive deposits. Géographie physique et Quaternaire, $35:$ : 301-316.

Carter, D., 1989. Réévaluation de la charge sédimentaire totale de la Grande Rivière et action des vagues sur la prise en charge des sédiments. Rapport final présenté à la SEBJ.

D’Anglejan, B., 1980. Effects of seasonal changes on the sedimentary regime of subarctic estuary, Rupert Bay. Sedimentary Geology, 26 : 51-68.

1994. Potential sedimentological impacts of hydroelectric development in James Bay and Hudson Bay. James Bay Publication Series, North Wind Information Service, Montréal, 2 : 1-15.

Dionne, J.-C., 1976. L'action glacielle dans les schorres du littoral oriental de la baie James. Cahiers de Géographie de Québec, 20 : 303-326.

1978. Le glaciel en Jamésie et en Hudsonie. Géographie physique et Quaternaire, $32: 3-70$.

Dyke, A.S., Vincent, J.-S., Andrews, J.T. Dredge, L.A. et Cowan, W.R., 1989. L'inlandsis laurentidien : introduction à la géologie quaternaire du Bouclier canadien, p.188-202. In R. J. Fulton, édit., Le Quaternaire du Canada et du Groenland. Commission géologique du Canada, Géologie du Canada.

Gostelow, T. P., 1996. Landslides, p.183-230. In V. P. Singh, édit., Hydrology of Disasters. Water Science and Technology Library, Netherlands.

GEL (Groupe Environnement Littoral Inc.), 1992. Géomorphologie littorale de la baie de Rupert et des côtes adjacentes. Rapport présenté à HydroQuébec, Vice-présidence Environnement, 73 p.

Guimont, P. et Laverdière, C., 1983. Le glaciel du lit asséché du Caniapiscau - du lac Duplanter au canyon Eaton. SDBJ, Aménagement régional, $106 \mathrm{p}$.

Hardy, L., 1977. La déglaciation et les épisodes lacustre et marin sur le versant québécois des basses terres de la baie de James. Géographie physique et Quaternaire, $31: 261-273$.

Hydro-Québec, 1990. Complexe Nottaway-Broadback-Rupert. Renseignements généraux, $47 \mathrm{p}$.

Hydrotech Inc., 1977. Aspects physique, hydraulique et morphosédimentologique des rivières Nottaway, Broadback et Rupert. Complexe NBR. Rapport présenté à la Société d'Énergie de la Baie James, 86 p.

1988. Problématique générale d'érosion et de sédimentation. Complexe NBR. Comité ADHOC, document de travail présenté à HydroQuébec, $65 \mathrm{p}$.

1991. Synthèse morpho-sédimentologique. Prédictions des bilans sédimentaires des rivières Nottaway, Broadback, Rupert et leurs tributaires et de la baie de Rupert. Complexe NBR, avant-projet Phase I, Rapport complémentaire (préliminaire), rapport présenté à Hydro-Québec, $41 \mathrm{p}$.

Knighton, D., 1998. Fluvial Forms \& Processes. A New Perspective. Oxford University Press, Don Mills, $383 \mathrm{p}$.

Laverdière, C. et Guimont, P., 1975. Le milieu bio-physique de la baie de Rupert. Société de développement de la Baie James, Direction de l'Environnement, $158 \mathrm{p}$.

1979. Le lit asséché de la Grande Rivière entre LG1 et LG2. Société de développement de la Baie James, Environnement et aménagement du territoire, rapport de reconnaissance, $34 \mathrm{p}$.

1980. Le lit asséché de la Grande Rivière. Ministère de l'Énergie et des Mines et Ressources, Canada, GEOS, p. 14-20.

Lawson, D.E., 1985. Erosion of Northern Reservoir Shores, an Analysis and Application of Pertinent Literature. US Army Corps of Engineers, Cold Regions Research and Engineering Laboratory, National Technical Information Service (CRREL, Monograph 85-1), 207 p.

Lupien, Rosenberg et Associés, 1991. Étude des berges de la rivière Broadback. Complexe NBR. Volume I, texte et annexes, rapport S-902337-3, présenté à Hydro-Québec, 82 p. 
1992. Complexe NBR, études complémentaires des berges de la rivière Broadback. Rapport S-91-2438-1, présenté à Hydro-Québec, 28 p.

Mollard, J.D. et Janes, J.R., 1985. La photo-interprétation et le territoire canadien. Approvisionnements et Services Canada, 424 p.

Poly-Géo Inc., 1991. Photo-interprétation et cartographie des grandes classes de dépôts de surface. Complexe Nottaway, Broadback et Rupert, Avantprojet Phase I. Rapport présenté à Hydro-Québec, Vice-Présidence Environnement, dossier 90120, 24 p.

SEBJ (Société d'énergie de la baie James), 1978. Connaissance du milieu des territoires de la Baie James et du Nouveau-Québec. Service Environnement, $297 \mathrm{p}$.

1982. Rapport préliminaire d'impact sur l'environnement et le milieu social. Description du territoire et de son environnement. Complexe NBR, volume 2, pour Hydro-Québec, décembre, 283 p., 19 pl.

1984. Comité de spécialistes sur les argiles sensibles du Complexe NBR. Vice-présidence, Ingénierie et développement, Service Géologie et Mécanique des sols, Rapport final, $158 \mathrm{p}$.

1991. Dynamique des berges de La Grande Rivière. Caractérisation de l'état de référence avant l'exploitation des centrales de La Grande 2A et de La Grande 1 (1989). Service Géologie et Mécanique des sols et SEBJ, $19 \mathrm{p}$.

Sogeam Inc., 1980a. Aménagements correctifs des tronçons aval des rivières Nottaway-Broadback-Rupert: validation sur le plan géomorphologique. Rapport présenté à Hydro-Québec. 1980b. Étude de la morphologie littorale de la baie de Rupert et évaluation des impacts selon les variantes d'aménagement. Rapport présenté à la SEBJ, $71 \mathrm{p}$.

Sogeam-Tecsult Inc., 1991. Complexe Nottaway-Broadback-Rupert, Avantprojet phase I, Études géomorphologiques, rapport de terrain et description du milieu. Rapport présenté à Hydro-Québec, Vice-présidence Environnement, 30 p. + cartes.

1992. Analyse morpho-sédimentologique des berges actuelles des lacs et rivières du Complexe NBR. Rapport présenté à Hydro-Québec Vice-présidence Environnement, Montréal, vol. 1, 290 p., vol. 2, cartes et graphiques.

1993. Complexe Nottaway-Broadback-Rupert, Avant-projet phase I, Études géomorphologiques. Morpho-sédimentologie et dynamique des berges actuelles des zones affectées et des berges futures du Complexe NBR. Rapport synthèse présenté à Hydro-Québec, Vice-présidence Environnement. 111 p. + cartes.

Sylvester, R., 1974. Coastal Engineering II. Elsevier, Amsterdam, 338 p.

Thorne, C. R., 1982. Processes and Mechanisms of River Bank Erosion, p. 227-259. In R.D. Hey, J. C. Bathurst et C.R. Thorne, édit., Gravel-bed Rivers. John Wiley, Chichester.

Vincent, J.-S., 1989. Le Quaternaire du sud-est du Bouclier canadien, p. 266295. In R.J. Fulton, édit., Le Quaternaire du Canada et du Groenland. Commission géologique du Canada, Géologie du Canada. 